\title{
Distinguishing on Applicable Scope and Degree of Soft Law under Chinese Context
}

\author{
Han Yi \\ Hohai University School of law, No. 1 Xi Kang Road, Nanjing, China \\ hanyi504@sina.com
}

Key words: soft law; soft law governance; public governance

Abstract: soft law governance has been considered as an important way to meet public governance demand and strengthen legal construction. But in current China, soft law is confronted with multiple problems on theory and system: when theoretical framework is constructed for soft law governance, elaborate design of system and rule is ignored and separated; when positioning and evaluation on soft law governance are performed, only tool-like understanding is realized and follow-up of social governance idea and governance capacity is ignored; when soft law is applied, relevant soft law governance scope and limit is not clarified correctly. In terms of modernization progress of legal system of China, soft law governance is necessary and feasible, and it is indeed demand of era to provide idea and system space for existence and positioning of soft law governance.

Soft law is not new phenomenon, but in new era, it has been generally accepted in worldwide public governance. ${ }^{[1]}$ Soft law does not take coercive force of the state as implementation guarantee, but it is not necessarily invalid completely. In other words, in terms of efficiency, although soft law is "soft", it is a kind of "rule phenomenon", and it has "binding force" or "toughness". "Toughness" of soft law comes from self-discipline and it also relies on consideration of self-interest and drive of social pressure, which distinguishes it from morality or inside guidelines.

Soft law has "toughness" and "elastic force", being an "uncertain concept". In reality, definition on soft law does not clarify adjustment scope and degree of its "toughness" or "weak efficiency"; it would be better to call the definition as rough summary and general term of existing rule phenomenon instead of "clarification of concept and elaborate re-creation". The fuzzy understanding may cause "rebound" of soft law easily: on the one hand, the adjustment scope may be extended to pure private sphere not concerned with public life, thus causing moralization of soft law where its legitimacy is varnished with traditional custom or even name of "goodness"; on the other hand, it may be difficult to distinguish implementation field of soft law and hard law, and still logic of public domain that "replace hard law with soft law" may be common.

If we pay more attention to social function analysis of soft law and "necessity" in initial research stage of soft law governance, then in creation and implementation phase of soft law, what needs to be cared urgently is "feasibility" of soft law governance. Especially under Chinese context, clarification effort on soft law concept 
and delicate design to soft law mechanism on the basis of it are crucial. Determination of applicable scope and degree of soft law is core problem to clarify soft law concept, and it helps soft law to evade cognition situation of "nonexistence of efficiency" or "being confused with hard law".

\section{Rise of soft law governance and reflection under Chinese context}

Compared with natural generation of public governance idea and pattern in the west, ${ }^{[2]}$ it would be better to call soft law governance as "rigid docking" instead of "demand of era" in China. Growth situation of public domain and difference with the west on traditional concept push numerous practical problems to the government of China and society in social management system reform process: lack of liberal tradition and self-government spirit, breeding of rule of law not experiencing long-term concept brewing, civil society being in initial development stage and state-centrism not disappearing completely.

Seen from development of government-governing mode, cognition and governance process of negation of negation in western countries from "the minimum government" to "omnipotent government" and to "multi-element public governance" is not experienced, and reflection experience of theory and practice is not enough relatively. Under effect of traditional custom and folk common law of China, soft law concept with multiple different meanings becomes more unpredictable. Although "law is re-systematism of habit" to some extent, excessive emphasis to "ethicality" of soft law may leave greater space for "rebound" of soft law. Confronted with complexity of soft law concept, changing from "objective sweeping generalization description" to "construction and re-creation of this regulation means in idea” is demand of public domain governance and the demand to realize good governance.

\section{Chinese-style prospect of soft law governance}

Free spirit and citizen governance are two spiritual cores of soft law. It takes individual as starting point and the essence is "self-legislation", which embodies respect to individual will and choice and recognition to self-discipline management of social organization; at the same time, it manifests brilliant rays of general will in value orientation, extruding communication interaction in procedure and understanding and support of participant. People place great hopes on soft law and it also plays an important role in social management system reform of China. Expectation exists in soft law governance: i.e.: disadvantage of hard law is corrected and remedied, the blank of hard law is filled, and detail of hard law is enriched through soft law specification to extend legalization domain and meet demand of national administration and social self-governance; support rational soft law practice through theoretical improvement and realize effective regulation of principle of rule of law and spirit of rule of law to relevant system arrangement. ${ }^{[3]}$

In practice, it seems that soft law governance is aimed at solution in "violation of constitution in good faith" directly - legalize error-trial decision "with no basis on law" hastily, and "abide by" the third way "stiffly" not belonging to "legal text 
specification". Compared with advantage of hard law on social cost saving, strengthening of multiple subject cooperation and social common interests with assistance and compensation of soft law to hard law makes soft law play a role not worse than that of hard law in public domain governance. But theory and practice "contradiction" of soft law is not solved because of it, and soft law causes a series of problems when it plays a role.

The first problem: unification of objective social function and internal value pursuit of soft law.

Form function and internal value spirit of soft law are separated to a certain extent, and citizen participation and democratic management spirit are not injected to the whole process of soft law governance necessarily. Even if soft law gets rid of formulation condition of government sector monopoly, double deficiency of independence capability of community organization and governmental responsibility may bring greater risk to soft law.

The second problem: unification of social objective and social effect of soft law governance.

Because it is difficult to quantize judgment standard and basis of social effect of soft law implementation, subjectivism in creation process of soft law is criticized and doubted, and so-called "flexibility" and "public welfare" of soft law will become "virtual advantage" difficult to be grasped. Let alone effect that may be caused by negotiation ability of the public and professional literacy factor on quality and evaluation of soft law.

\section{Distinguishing on scope and degree of soft law}

Determination of scope and degree of soft law shall be developed by combining with specific condition and specific problem of China. Incompatibility of idea tradition and special pattern development experience of rule of law increase difficulty of concept clarification of soft law. Because of separation of objective social function and internal value pursuit of soft law, it is difficult to avoid the behavior of "replacing value with form”, and "implementing nationalization in the name of localization". Under the background where it is difficult to judge social effects of soft law governance, even if the idea that "government shall choose soft law instead of hard law as public governance means firstly when the same social goal is realized" is to be built, it is difficult to gain the opportunity of "choosing soft law firstly" or "replacing hard law (soft law)" in practical operation.

To realize "placement" of adjustment scope and degree of soft law, in adjustment scope of soft law, boundary shall be decided with "publicity" and "socialization”. The limit avoids excessive intervention of soft law to morality adjustment domain substantially - such as content of subjective will involving emotion relationship of relatives and friends in private sphere.

Secondly, look at substantial contents reached through consensus in degree to avoid the behavior of over-distention of moral level by soft law in the name of "goodness" and limiting free action space of individual. After all, in universal meaning, it can be said that "goodness" is common ultimate pursuit and measurement 
index of code of human conduct and ethical value of hard law or soft law can be questioned closely.

Moreover, distinguishing hard law and soft law by taking "whether it can guarantee implementation by replying on coercive force of the state" as watershed is not comprehensive, and that all rules not belonging to hard law are called as "pan-soft-law" makes soft law become a meaningless "pocket" concept. In fact, soft law shall be "rule that exists actually and that can form effective behavior restriction" at least. "Soft" specification in hard law that has weak coercive force of the state and that is difficult to be implemented shall be distinguished from soft law. Eliminating hard law specification with strong moral significance directly and endowing it the "identity" of soft law will not help to solve the awkwardness of its internal content in necessity and exploitativeness, but impress people with "mere formality" and "bad rule” on soft law.

\section{Conclusion}

Soft law is not all-powerful. Soft law may not adjust scope that is not reached by hard law necessarily. Even if soft law has permeated to every aspect of life, social contradictions may not disappear at this point necessarily. Except for applicable space of hard law, application of soft law may not represent approaching of substantial justice and goodness necessarily. No matter cognition positioning performed to soft law from social function and effect or macroscopic definition to system category of soft law from theoretical perspective, follow-up of internal idea spirit of soft law is the key. In practice, elaborate design to relevant system and rule cannot be replaced by "packaging" classified recognition. On the other hand, when people define some rule forms as soft law, purpose of people is not merely to improve soft law theory with it, and to look forward to consider these rules as evidence of social governance mode transformation more.

It thence appears that importance of soft law research does not merely lie in macroscopic construction of its theoretical system and extraction of generality of the rule, but also lies in content legitimacy measurement to system resource incorporated to soft law, such as public policy and self-discipline specification etc. and detailed research on connection of soft and hard law. Correspondingly, positioning of soft law in social governance structure cannot be confined to simple conclusion of the right and the wrong and the good and the bad, but it shall find optimal portfolio between specific domain and specific way of various public institutions on the basis of systematical experiential knowledge.

\section{References}

[1] Refer to Zhai Xiaobo: Soft Law Concept and Public Governance, quoted from Soft Law and Public Governance of Luo Haocai et al., Peking University Press, 2006, pp. 144.

[2] Public governance is not government rule means again, but representing new social multi-element management mode, and some scholars generalize it as "open 
public management and wide public participation”. Refer to Public Governance and Public Ethic of Li Jianhua et al., Hunan University Press, 2009, pp. 1-3; Luo Haocai and Song Gongde: Transformation of Public Domain Governance, quoted from Soft Law and Public Governance of Luo Haocai et al., Peking University Press, 2006, pp. 21.

[3] Refer to Soft Law Also Being Law: Public Governance Needing Soft Law Governance of Luo Haocai and Song Gongde, Law Press, 2009, pp. 4. 